\title{
Comprehensive Identification of the Particulate Matter Population Present in Medical Products and their Manufacturing Processes
}

\author{
X. Gu${ }^{1 *}$, J.R. Mantei ${ }^{1 *}$ \\ 1. Baxter Healthcare, Pharmaceuticals R\&D, Round Lake, IL, USA. \\ * Corresponding author: xin_gu@baxter.com; jason_mantei@baxter.com
}

The presence of particulate matter (PM) in medical products and manufacturing processes is a wellestablished and accepted fact. Regulators have strict rules regarding the size distribution and number of particles that are allowed in medical products. Therefore, understanding and controlling the PM in manufacturing processes and their resultant products becomes a crucial task for the healthcare and pharmaceutical industry. In this work, we introduce a method that can provide full characterization of the PM population present in a medical product or manufacturing process, ranging from aggregate measures of the total number of particles and their size distribution to data on each individual particle, including the size, shape, and most importantly, chemical and elemental composition. Such information gives valuable, statistically unbiased insight into the full PM population, and helps to pinpoint and identify strategies to eliminate the primary sources of the PM in an accurate and proactive manner if necessary.

In this method, we utilize a suite of techniques including optical microscopy, image segmentation, spectroscopy (Raman, FTIR, EDXS), and multivariate analysis to investigate the PM of interest. With a combination of inherent instrument software functionality and customized MATLAB code, these analyses are largely automated, leading to the ability to identify more than 1,000 particles in a matter of days with minimal analyst involvement.

Specifically (Figure 1), fluid containing representative PM population is filtered through a gold coated membrane. Multiple units of the same type can be filtered or rinsed in order to increase the number of particles deposited on the membrane. A self-designed, 3D-printed membrane lock with 4 built-in fiducial marks is then applied to immobilize the membrane onto a petri slide. An automated optical microscope images the entire membrane and the data is segmented to acquire the size, shape, and coordinates of all PM on the membrane. Subsequently, using our coordinate conversion algorithm, particles can be accurately located on each analytical instrument, and are subjected to Raman, FTIR, and EDXS measurements in an automated fashion.

Flexible solution containers filled with unfiltered water, simulating an intravenous therapy product (which were filtered as-is), as well as test fluid transfer sets (which were rinsed with particle-free water, which was then collected and filtered) were chosen as examples to demonstrate the feasibility and power of our method. After completion of the analysis, a snapshot of the full PM pareto specific to each individual test system is known (Figure 2 (a) - (d)). This data has the potential to greatly enhance our understanding regarding the PM in our products and processes. By studying a particular system over time, assessing before-and-after PM burden when changes are made to a process, looking at similar processes performed in different global locations, etc., process improvements and preventative actions can be performed to reduce PM-related issues in the pharmaceutical industry. 


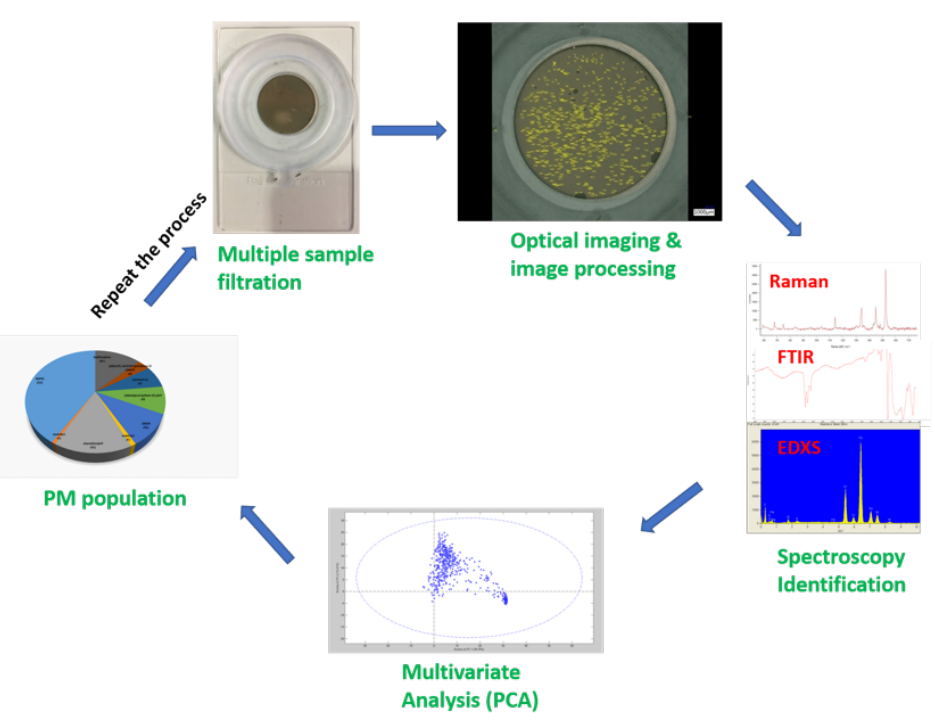

Figure 1. PM characterization flow chart.

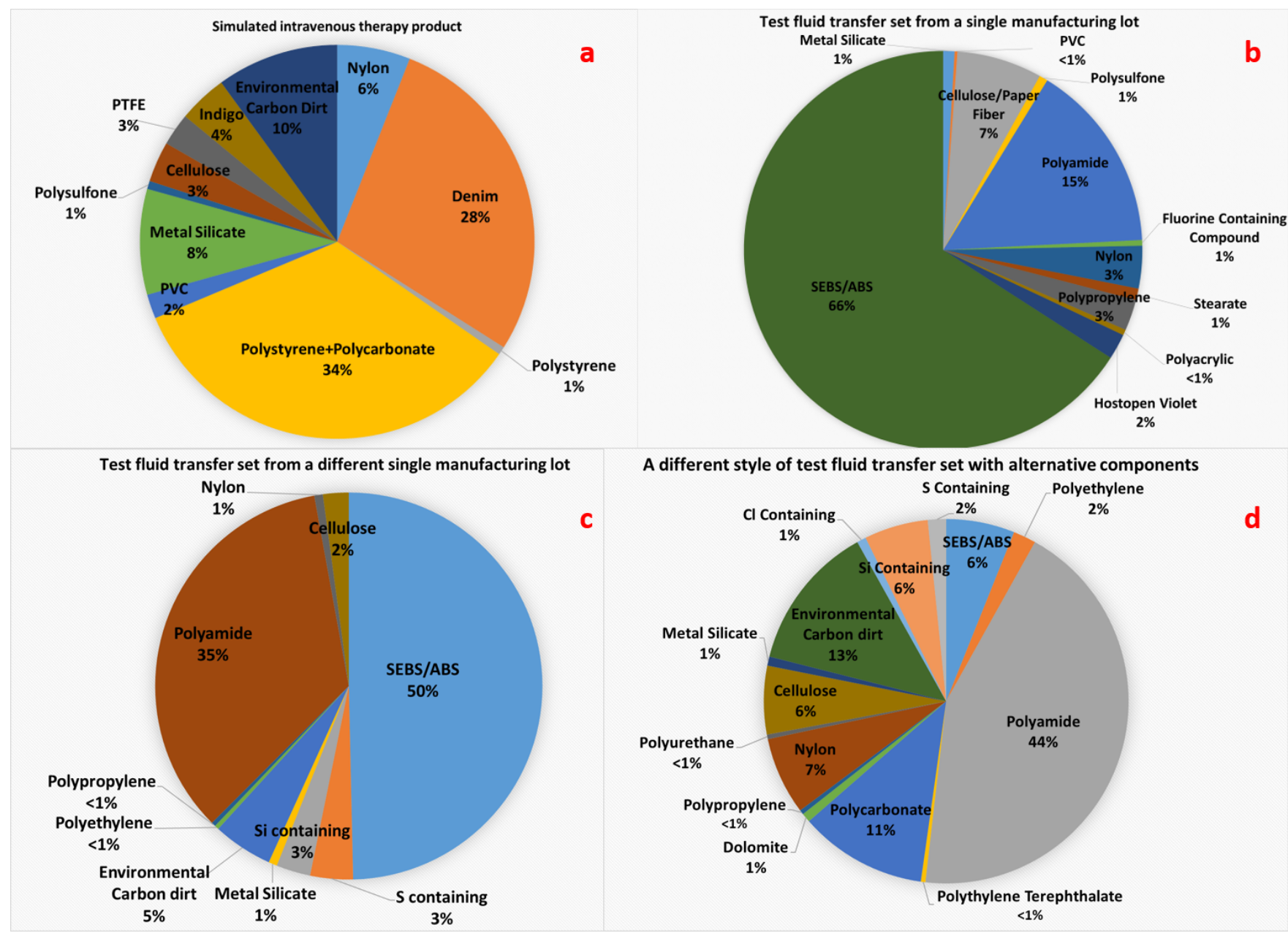

Figure 2. PM population of (a) simulated intravenous therapy product in which flexible containers were filled with unfiltered water, (b) test fluid transfer set from a single manufacturing lot, (c) test fluid transfer set from a different single manufacturing lot, (d) a different style of test fluid transfer set containing alternative components. 